\title{
ECC
}

\section{Study of bendamustine anticancer drug in gaseous and in a few selected liquid solvents phases using functional density theory (DFT)}

\author{
Mehri Deilam, Ashraf Sadat Ghasemi*, Fereydoun Ashrafi \\ Department of Chemistry, Payame Noor University, P.O. BOX 19395-3697, Tehran, Iran
}

Received: 19 April 2019, Accepted: 9 June 2019, Published: 1 November 2019

\begin{abstract}
In this research, bendamustine anticancer drug in main and excited states in gaseous phase and in water and ethanol and methanol solvent phases have been studied, using DFT at MPW1PW91/6-311++G(d, p) theoretical level. The molecules of solvent affected the equilibrium structure of species. The effects of solvents have been studied by polarized continuum model (PCM). The optimized structural parameters in gaseous and in water, ethanol and methanol solvent phases as frequency data, relative energy, dipole moment, the energy of highest occupied molecular orbital (HOMO), the energy of lowest unoccupied molecular orbital (LUMO), density functional graphs and gap energies have been calculated. The results of the theoretical computations confirm that the structure of drug, in both the main and the excited states, is more stable in liquid phase than in gaseous phase, and most stable in water. The computation results confirm that the drug stability increase by increasing in polarizability of the solvent.
\end{abstract}

Keywords: Bendamustine; gaseous and liquid phases; main state; excited state; polarizability.

\section{Introduction}

The exact cause of asymmetric division of body cells namely cancer is unknown. Probably some genetic factors or some cases that perturb the activity of the cells can create the problem in cells core that may be the origin of cancer [1]. Abnormal growth of one cell can cause abnormal growth of other cells and finally results in cancer [2-5]. Nowadays, chemotherapy is the most important treatment of cancer. Chemotherapy, for the first time, has done by one of the existing drugs in 1955, but nowadays much more drugs are used for this case [6,7]. Now, chemotherapy is a common method of cancer treatment that can destroy cancer cells [8]. In this research, bendamustine anticancer drug has been investigated by theoretical and computational methods. Bendamustine drug (BENDA) was firstly synthesized in 1963 in East Germany by Ozegowski and Krebs [9, 10]. This drug has the trade names Treanda, Treakisym, Ribomustin, Levact and other drugs have been used in the cancer treatments as malignant tumors, chronic lymphocytic leukemia (CLL), multiple myeloma, nonHodgkin's lymphoma, and lung cancer, by injection into a vein $[11,12]$. 
Bendamustine belongs to the alkylating medications family. This drug also has been investigated for sarcoma treatment [10].

DFT method as a useful and assured method has been exerted to compute electronic structure theory and spectroscopic properties of different types of compounds [6]. This method has been the most common method used in Computational Chemistry in the last ten years $[13,14]$. This method has been used in much more studies as interaction of molecules and adsorption effects of different molecules and elements on the different surfaces [15-29]. Many authors also, in order to understand the biological and anticancer activity of certain drugs inevitably, have studied the physicochemical properties using DFT method [30-41].

In this paper, the basic and excited states of Bendamustine drug have been studied in the gaseous and in the liquid solvents phases using DFT method at MPW1PW91 theoretical level (for basic state) and at MPW1PW91-TD theoretical level (for excited state) at 6$311++\mathrm{G} \quad(\mathrm{d}, \mathrm{p})$ basis orbital set. Furthermore, thermodynamically properties, relative energy, dipole moment, structural parameters, gap energy and HOMO and LUMO molecular orbitals have of the drug been investigated and discussed.

\section{Computational methods}

In this study, the structure of Bendamustine drug has been optimized using DFT method (MPW1PW91 for main state and MPW1PW91-TD for excited state) performing $6-311++\mathrm{G}$ $(d, p)$ basis set. The effects of solvent on the main and excited states have been performed by polarized continuum model (PCM) [42]. Polarity of the solvent has been measured by dielectric constant or relative permittivity. As much as the dielectric constant is higher, the solvent polarity is higher. Modeling the effects of solvent, PCM that belongs to the classification of polarized solvents model has been used. In this model, the solvent considered to be a continuum polarization instead of individual molecules, can use for different systems [43-48]. PCM model also studies the stability and energy of structures [49]. The density of states has plotted at this level of theory to obtain the gap energy [50]. Quantum mechanics calculations have been performed by Gaussian 03 software [51]. To obtain more details, the electrophilicity index $(\omega)$ has been calculated. This index measures the value of system stability during charge transfer. The magnitude of $\omega$ indicates the stability of structure [52]. Chemical potential $(\mu)$ and Chemical hardness $(\eta)$ also have been calculated to obtain more details. The above mentioned indexes have also been calculated using following equations:

$$
\begin{aligned}
& \mu=-\frac{(I+A)}{2} \\
& \eta=\frac{(I-A)}{2} \\
& \omega=\frac{\mu^{r}}{r \eta}
\end{aligned}
$$

Where I (-Eнмо $)$ is ionization potential and A (-E EUMO) is electron-affinity. Calculating Eномо and Elumo the gap energy, $\mathrm{E}_{\mathrm{g}}$ has obtained.

\section{Results and discussions \\ Optimization of structures}

Quantum mechanics computations have been performed using Gaussian 03 software. The structural parameters of Bendamustine drug have been optimized using MPW1PW91 method and at $6-311++\mathrm{G}(\mathrm{d}, \mathrm{p})$ basis orbital set. Also, the frequencies computations have been carried out to study vibration techniques and zero point corrections at 
above mentioned computation level. Time-dependent density functional theory (TD-DFT) has been applied for the excited state. The optimized

structure of Bendamustine drug using above mentioned computations, is shown in Figure 1.

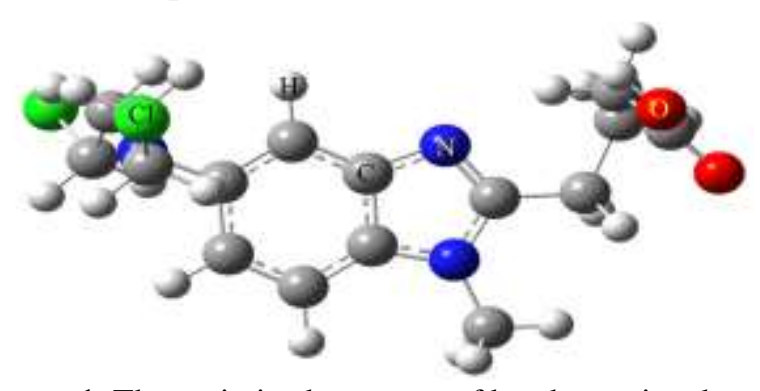

Figure 1. The optimized structure of bendamustine drug

Structure and binding energy

The relative energy, $\mathrm{E}$, the relative energy differences, $\Delta \mathrm{E}$, and dipole moment have been calculated in the ground and excited states in the gaseous phase and in three solvents (ethanol, methanol and water) at MPW1PW91/6$311++\mathrm{G}(\mathrm{d}, \mathrm{p})$ quantum chemical level and have shown in Table 1.

Table 1. The relative energy, E, the relative energy differences, $\Delta \mathrm{E}$, and dipole moment computed in the ground and excited states in the gaseous phase and in three solvents

\begin{tabular}{lcccccc}
\hline \multicolumn{1}{c}{ Phase } & \multicolumn{3}{c|}{ Ground state } & \multicolumn{3}{c}{ Excited state } \\
& $\mathbf{E}(\mathbf{e V})$ & $\mathbf{\Delta E}(\mathbf{e V})$ & $\mathbf{D}(\mathbf{D e b y e})$ & $\mathbf{E}(\mathbf{e V})$ & $\mathbf{\Delta E}(\mathbf{e V})$ & $\mathbf{D}(\mathbf{D e b y e})$ \\
\hline Gas & -50547.202 & 0.603 & 2.73 & -50547.006 & 0.726 & 2.10 \\
Ethanol & -50547.776 & 0.029 & 3.29 & -50547.702 & 0.030 & 1.95 \\
Methanol & -50547.786 & 0.019 & 3.30 & -50547.712 & 0.020 & 1.95 \\
Water & -50547.805 & 0.000 & 3.32 & -50547.732 & 0.000 & 1.92 \\
\hline
\end{tabular}

According to this Table, the stability of structure of drug in both ground and excited states decreases in the order of water>methanol>ethanol>gas. The results shown in Table 1 have shown that the stability of drug in both states increases with the increase in the polarity of solvent. Hence, the stability of molecule in liquid solvents is more affected by polarity variation than in gaseous phase. Also, among the selected solvents, it is more stable in aqueous phase.

The energy in water solvent has been calculated, in ground state it is $50547.805 \mathrm{eV}$ and in excited state it is $50547.732 \mathrm{eV}$. Therefore, in aqueous phase, the drug is more stable in ground state than in excited state.
As seen in Table 1, the dipole moment of drug in ground state has increased from gaseous phase to solvent phase. So it is expected that the stability of molecule in ground state increase with the increase in polarity of solvent. Whereas, the variation of dipole moment in excited state is inverse, comparing to the ground state. So that, the dipole moment is grater in ground state and in aqueous phase.

Electronic energies and the relative stability

The highest occupied molecular orbitals (HOMO) and the lowest unoccupied molecular orbitals (LUMO) that are sometimes called frontier orbitals are very important parameters in quantum chemical studies. As much as the energy of HOMO orbitals is greater, it loses the 
electron more easily, and as much as the energy of LUMO orbitals is smaller, it accepts the electron more easily [53]. The HOMO and LUMO orbitals of Bendamustine drug in ground states and in excited states in gaseous phase and in different solvents have been shown in Figures 2 and 3. The difference between HOMO and LUMO energies has called gap energy $\left(\mathrm{E}_{\mathrm{g}}\right)$. Studying the specifications and the characteristics of these orbitals and their energy differences could lead to better understanding and the clarification of kinetic stability, chemical reactivity of molecules and interamolecular charge transfer. As seen in Figure 2, a HOMO distribution in the ground state, on the molecules of the drug is asymmetric, and in the excited state, a greater part of the molecule has covered by the distribution of HOMO. This may be attributed to the contribution of more orbitals in excited state. The distribution of LUMO orbitals remains approximately the same in the both ground and excited states.

(a)
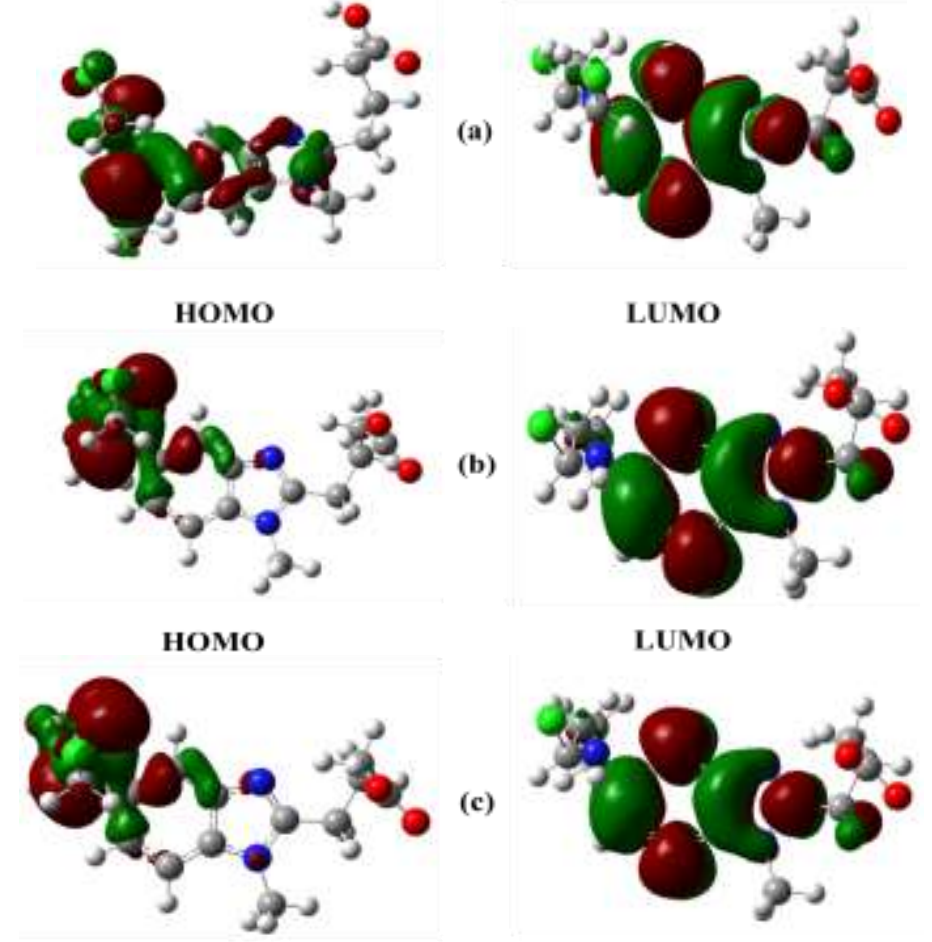

номо

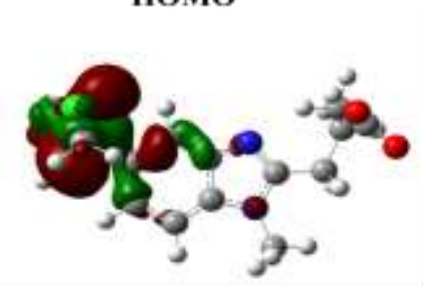

Hомо
LUMO

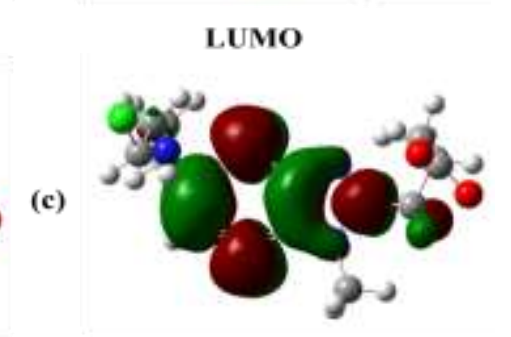

LUMO

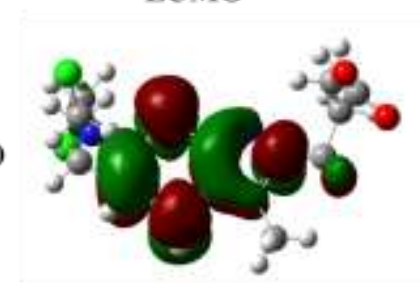

LUMO

Figure 2. HOMO and LUMO molecular orbitals of bendamustine drug in ground state in (a) gaseous, (b) ethanol, (c) methanol and (d) aqueous phases 


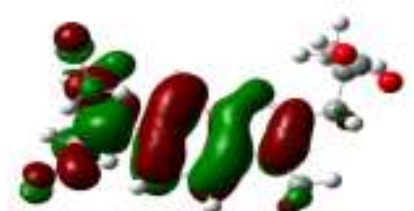

HOMO

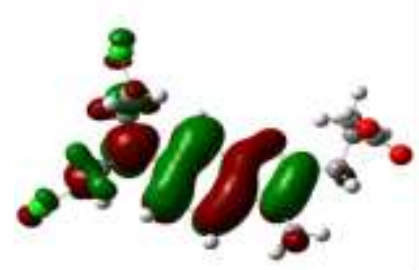

HOMO

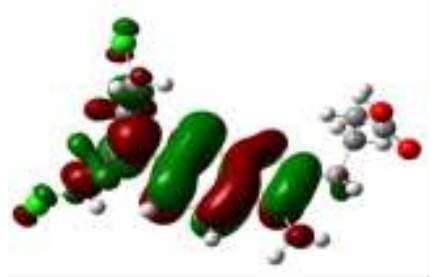

HOMO

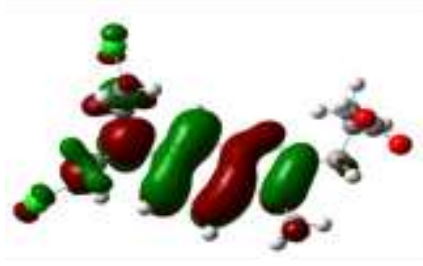

HOMO (e)

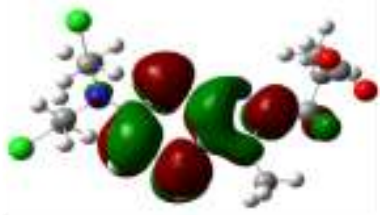

LUMO

(f)

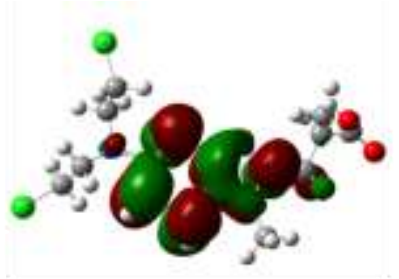

LUMO

(g)

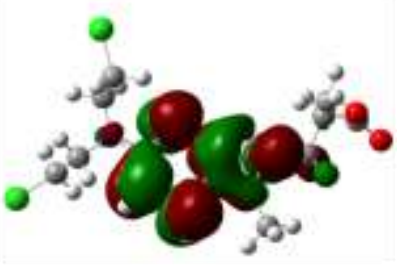

LUMO

(h)

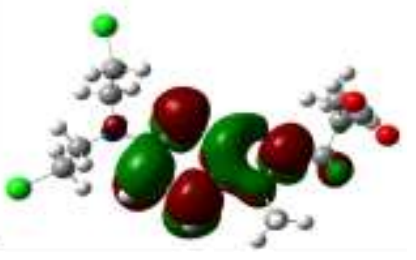

LUMO

Figure 3. HOMO and LUMO molecular orbitals of bendamustine drug in excited state in (e) gaseous, (f) ethanol, (g) methanol and (h) aqueous phases

The density of states plots (DOS) have shown in Figures 4 and 5 that indicate well the level of energies in

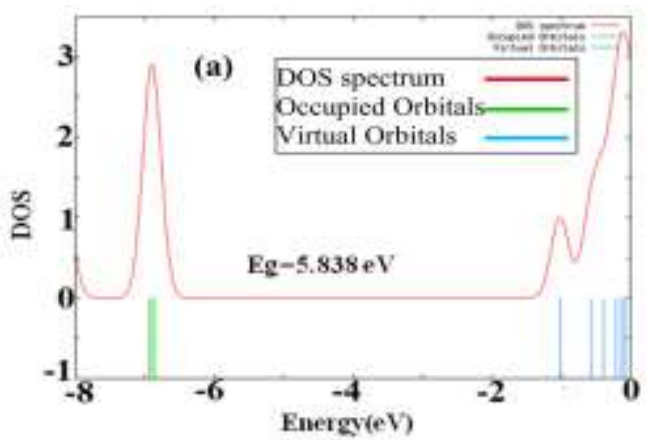

HOMO and LUMO orbitals as well the gap energies.

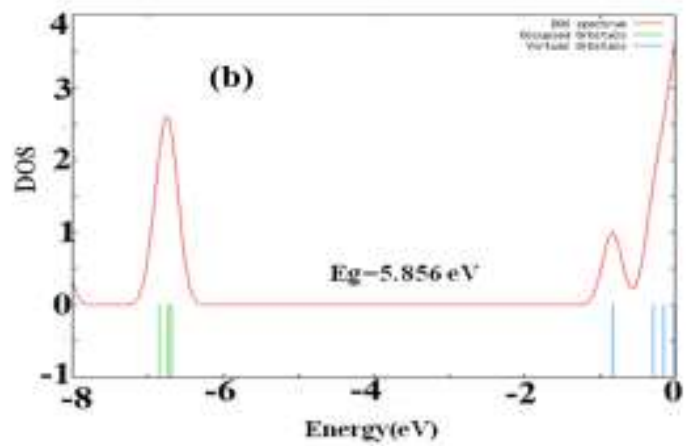



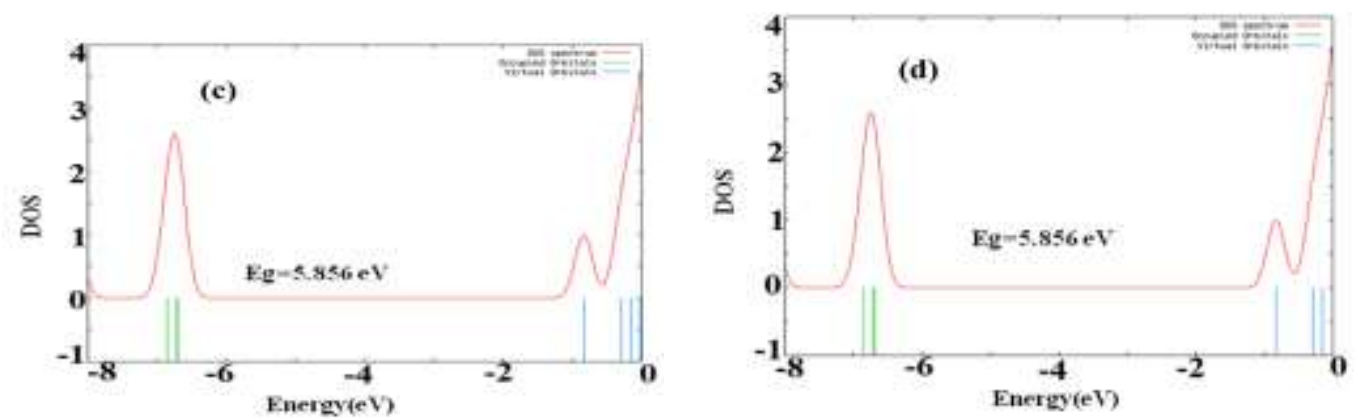

Figure 4. DOS plots of bendamustine drug in ground state in (a) gaseous, (b) ethanol, (c) methanol and (d) aqueous phases
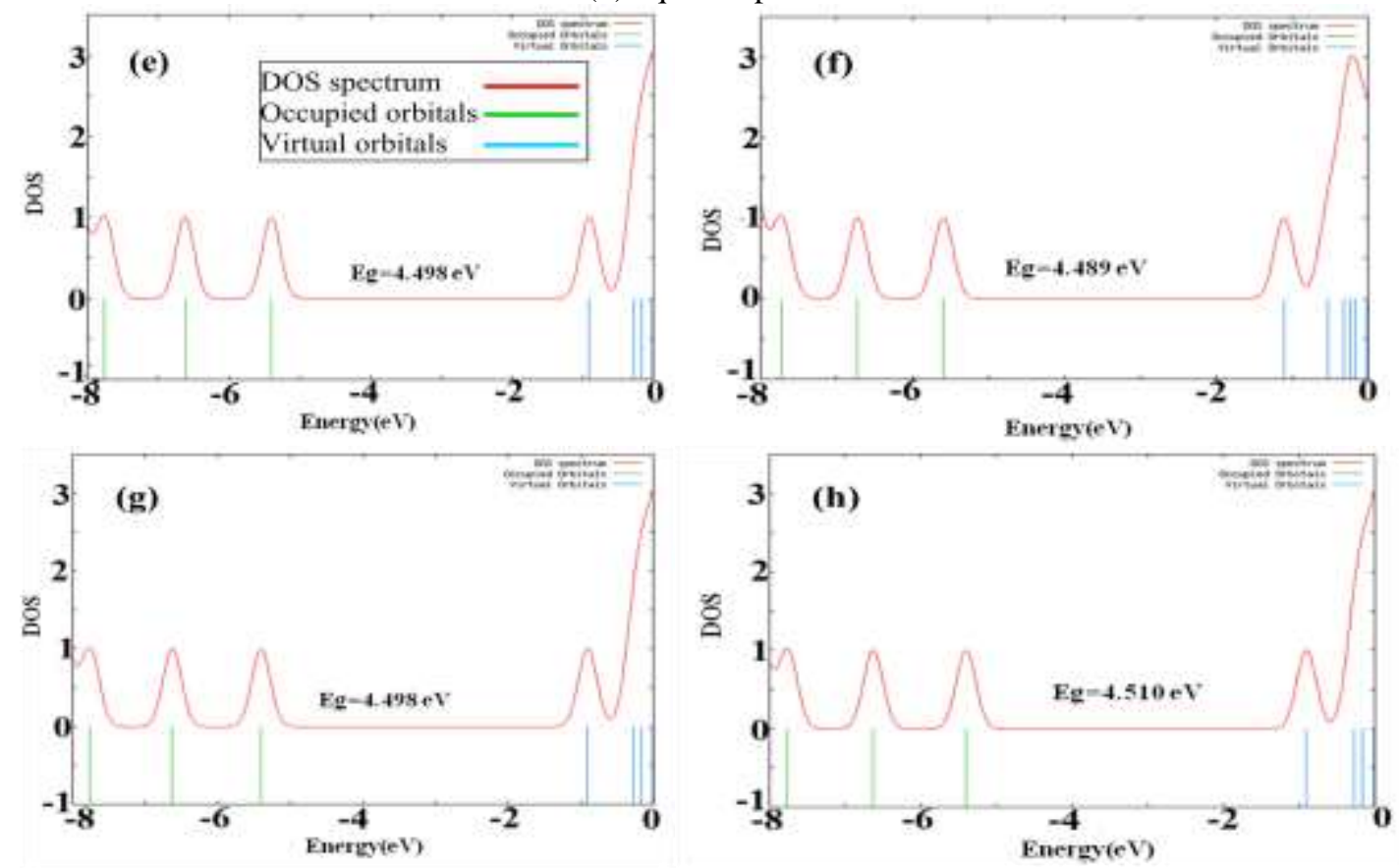

Figure 5. DOS plots of bendamustine drug in excited state in (e) gaseous, (f) ethanol, (g) methanol and (h) aqueous phases

The results of computations at MPW1PW91 level and 6-311++G(p,d) basic orbital set of HOMO energy ( $\left.\mathrm{E}_{\text {HOMO }}\right)$, LUMO energy ( $\left.\mathrm{E}_{\text {LUMO }}\right)$, gap energy $\left(E_{g}\right)$ and other quantum chemical properties as electrophilicity index $(\omega)$, chemical potential $(\mu)$, chemical hardness ( $\eta)$, I (-E $\left.\mathrm{E}_{\mathrm{LUMO}}\right)$ in ground and excited states have resumed in Table 2.

Table 2. The calculated values of $\mathrm{E}_{\mathrm{HOMO}}, \mathrm{E}_{\mathrm{LumO}}, \mathrm{E}_{\mathrm{g}}, \omega, \mu, \eta, \mathrm{I}$ and $\mathrm{A}$ of Bendamustine drug in ground

\begin{tabular}{lllll|llll} 
& \multicolumn{7}{l}{ and excited states in (a) gaseous, (b) ethanol, (c) methanol and (d) aqueous phases } \\
\cline { 2 - 9 } Phase & Ground state & \multicolumn{7}{c}{ Excited state } \\
\hline Unite & $\mathrm{eV}$ & $\mathrm{eV}$ & $\mathrm{eV}$ & $\mathrm{eV}$ & $\mathrm{eV}$ & $\mathrm{eV}$ & $\mathrm{eV}$ & $\mathrm{eV}$ \\
\hline EHomo & -6.853 & -6.683 & -6.681 & -6.679 & -5.593 & -5.404 & -5.403 & -5.402 \\
ELUMO $_{\text {LUM }}$ & -1.015 & -0.827 & -0.825 & -0.822 & -1.105 & -0.906 & -0.906 & -0.893 \\
$\mathbf{E}_{\mathbf{g}}$ & 5.838 & 5.856 & 5.856 & 5.856 & 4.489 & 4.498 & 4.498 & 4.510 \\
$\mathbf{I}$ & 6.853 & 6.683 & 6.681 & 6.368 & 5.593 & 5.404 & 5.403 & 5.402 \\
$\mathbf{A}$ & 1.015 & 0.827 & 0.825 & 0.822 & 1.105 & 0.906 & 0.906 & 0.893 \\
$\boldsymbol{\eta}$ & 2.919 & 2.928 & 2.928 & 2.973 & 2.440 & 2.249 & 2.249 & 2.255 \\
$\boldsymbol{\mu}$ & -3.934 & -3.755 & -3.753 & -3.595 & -3.349 & -3.155 & -3.155 & -3.148 \\
$\boldsymbol{\omega}$ & 2.651 & 2.408 & 2.405 & 2.330 & 2.298 & 2.213 & 2.213 & 2.197 \\
\hline
\end{tabular}


Studying the data shown in Table 2 reveals that the HOMO energies in gaseous and solvents phases in the ground state have located in lower levels as compared to those in the excited state. Whereas, in case of the LUMO energies, inversely to the HOMO energies, the orbitals in the excited state have located in lower levels comparing to those in the ground state. The variations of $\mathrm{E}_{\text {HOMO }}$ and ELUMO in both ground and excited states are in order of gas $<$ ethanol $<$ methanol <water that has indicated the greater stabilization of drug in aqueous phase. Furthermore, EHOMO in excited state in water has the smallest negative value $(-5.402 \mathrm{eV})$ which indicates the best electron-donation in the aqueous phase. Also, EHOMO in the ground and excited states in gaseous phase has the greatest negative value $(-1.015 \mathrm{eV})$ which indicates the best electronacceptation is in this phase.

The values of $E_{g}$ in all phases in the excited state are smaller than those in the ground state which indicate that the stability of drug in excited state is lesser and the reactivity is greater in this phase. The lowest $\mathrm{E}_{\mathrm{g}}$ in ground state is $5.836 \mathrm{eV}$ (in aqueous phase) and the lowest $\mathrm{E}_{\mathrm{g}}$ in excited state is $4.489 \mathrm{eV}$ (in gaseous phase).

The chemical hardness $\eta$, may be considered as a measure of the resistance of a chemical species against the changes of electron configuration [54]. The decrease in gap energy leads to decrease in chemical hardness. In the case of the Bendamustine drug in both ground and excited states the chemical hardness in gaseous phase is smaller than that in the solvents. The smallest amount of chemical hardness of the drug in excited state and in gaseous phase is $2.440 \mathrm{eV}$ with the smallest value of $E_{g}$.
The chemical potential $\mu$, may be considering as a measure of the system's tendency to lose its electron cloud [55]. In this study the chemical potential of the Bendamustine drug in both ground and excited states in gaseous phase is smaller than that in the solvents. The decrease in Eg, $\eta$ and $\mu$ leads to the decrease in stability and increase in the reactivity of the drug compared to the reactivity in solvents phases.

The electrophilicity index $\omega$, may be considering as a measure of the electrophilic potency of a chemical species. The greater electrophilicity index of the drug represents its higher electron affinity. In the case of the Bendamustine drug in both ground and excited states the electrophilicity index in gaseous phase is greater than that in the solvents phases. Finally, the electrophilicity index of all phases in the ground state is greater than that in the excited state.

\section{Conclusion}

In this study, the Bendamustine anticancer drug have been studied in the ground and excited states and in gaseous and some solvents phases. The obtained values of HOMO and LUMO energies have shown that the structure of drug in both ground and excited states and in the gaseous phase has more negative energy and thus has more stability as compared to the solvents phases.

Considering the values of HOMO and LUMO energies, $E_{g}$ in the excited one has decreased in all phases as compared to the ground state. Due to the decrease of Eg, the chemical hardness, chemical potential and electrophilicity have decreased and thus the stability of drug and its reactivity have been increased. As a result, the drug structure predominates in the ground state in the solvent phase. 


\section{Acknowledgements}

The authors thank Research Council of Payame Noor University for the financial support of this research.

\section{References}

[1] W.M. Becker, L.J. Kleinsmith, J. Hardin, G.P. Bertoni, Benjamin Cummings publisher, San Francisco, CA, 2009.

[2] B. Panchapakesan, S. Lu, K. Sivakumar, K. Teker, G. Cesarone E. Wickstrom, J. Nano. Bio. Tech., 2005, 1, 133-139.

[3] Cancer, World Health Organization, Retrieved on 11/12/2016.

[4] A.G. Cuenca, H. Jiang, S.N. Hochwald, M Delano, W.G. Cance, S.R. Grobmyer, J. Cancer, 2006, 107, 459466.

[5] Cephalon Receives FDA Approval for TREANDA, a Novel Chemotherapy for Chronic Lymphocytic Leukemia, Cephalon press release, Retrieved 2303-2008.

[6] K. Zare, N. Shadmani, E. Pournamdari, J. Nanostructure in Chemistry, 2013, 3, 1-6.

[7] (a) A. Yadav, V.K. Singh, J. Molecular Structure (Theochem), 1997, 389, 191-198; (b) E. Rezaee Nezhad, Z. Abbasi, S. Sajjadifar, SCIENTIA IRANICA, 2015, 22, 903-910.

[8] A.C. Dubbelman, H. Rosing, M. Darwish, D. D'Andrea, M. Bond, E. Hellriegel, P. Robertson Jr, J.H. Beijnen, J.H. Schellens, Drugs $R$ D., 2013, 13, 17-28.

[9] W. Ozegowski, D. Krebs, J. Prakt Chem., 1963, 20, 178-86.

[10] R. Kath, K. Blumenstengel, H.J. Fricke, K. Höffken (January 2001). J. Cancer Res. Clin. Oncol., 2001, 127, 48-54.

[11] S. Bagchi (August 2007). Bendamustine for advanced sarcoma. Lancet Oncol., 2007, 8, 674.
[12] R. Weide, G. Hess, H. Köppler; et al. Leuk. Lymphoma., 2007, 48, 12991306.

[13] F. Sen, O. Ekici, M. Dincer, A. Cukurovali, J. Molecular Structure, 2015, 1086, 109-117.

[14] A.S. Ghasemi, M. Deilam, J. Sharifi-Rad, F. Ashrafi, S.M. HoseiniAlfatemi, Cell. Mol. Bio., 2015, 61 110118.

[15] Soltani, M.T. Baei, A.S. Ghasemi, E. T. Lemeski, K. H. Amirabadi, Superlat. Microstruc., 2014, 75, 564575.

[16] A. Ghasemi, M. Molla , F. Ashrafi, Res. J. Appl. Sci. Eng. Tech., 2012, 4, 2543-2547.

[17] A.S. Ghasemi, M. Molla, Inter. J. Chem Tech Res., 2012, 4, 1302-1308.

[18] A.S. Ghsemi, F. Ashrafi, Res. J. of Appl. Sci. Eng. Tech., 2012, 4, 25232528.

[19] F. Ashrafi, A.S. Ghasemi, S.A. Babanejad, M. Rahimof, Res. J. of Appl. Sci. Eng. Tech., 2010, 2, 547-551.

[20] F. Ashrafi, A.S. Gasemi, E-J. Chem., 2012, 9, 2134-2140.

[21] S.A. Babanejad, F. Ashrafi, A. Ghasemi, Arch. Appl. Sci. Res., 2010, 2, 438-443.

[22] A.S. Ghasemi, F. Ashrafi, S.A. Babanejad, M. Rahimof, Arch. Appl. Sci. Res., 2010, 2, 262-270.

[23] A. Ghasemi, F. Ashrafi, Res. J. Appl. Sci. Eng. Tech., 2012, 4, 25292536.

[24] S.A. Babanegad, F. Ashrafi, A.S. Ghasemi, E. Ashrafi, Der Chem. Sin., 2012, 3, 124-128.

[25] A.S. Ghasemi, F. Aashrafi, Inter. J. ChemTech Res., 2012, 4, 1295-1301.

[26] A.S. Ghasemi, M. Rezaei, M. Molla, Inter. J. ChemTech Res., 2013, 5, 1585-1593.

[27] F. Ashrafi, S.A. Babanegad, A.S. Ghasemi, Res. J. Appl. Sci. Eng. Tech., 2012, 4, 795-801. 
[28] A.S. Ghasemi, Research Journal of Applied Sciences, Engineering and Technology, 2013, 5, 1892-1898.

[29] A.S. Ghasemi, M. Molla, M. Mostashregh, Inter. J. ChemTech Res., 2013, 5, 1623-1629.

[30] N.E. Mircescu, A. Varvescu, K. Herman, V. Chis, N. Leopold, Spectroscopy, 2011, 26, 311-315.

[31] F. Gökalp, Inter. J. Med. Medical Sci., 2014, 6, 146-150.

[32] H. Ghahremani, S. Bagheri, S.M. Hassani, Inter. J. Pharma. and Pharmac. Sci., 2012, 4, 751-754.

[33] H. Ghahremani, S.M. Hassani, Inter. J. PharmTech Res., 2012, 4, 130134.

[34] S.M. Hassani, S. Bagheri, Adv. in Biores., 2012, 3, 45 - 48.

[35] S.M. Hassani, S. Bagheri, Inter. J. PharmTech Res., 2012, 4, 1242-1246.

[36] S.M. Hassani, H. Ghahremani, S. Bagheri, Schol. Res. Lib., 2011, 3, 296300.

[37] S. Bagheri, Z. Chamani, S.M. Hassani, Inter. J. ChemTech Res., 2012, 4, 63-67

[38] F. Rahimi, A.Bahlake, Z. Chamani, S. Bagheri, Europ. J. Experim. Bio., 2012, 2, 2055-2060.

[39] J. Liao, H. Zhao, L. Zhou, Comput. Theo.l Chem., 2014, 1048, 84-94.

[40] (a) P. Sarmah, R.C. Deka, THEOCHEM, 2010, 955, 53-60; (b) S.

Rezayati, S. Sajjadifar, Journal of Sciences, Islamic Republic of Iran, 2014, 25, 329-337.

[41] O.V. Shishkin, A. Pelmenschikov, D.M. Hovorun, J. Leszczynski, J. Mol. Struc., 2000, 526, 329-341.
[42] D. Peer, S. Hong, O.C. Farokhzad, R. Margalit, R. Langer, Nat. Nanotech., 2007, 2, 751-760.

[43] M. Yoosefian, N. Etminan, S. Ahmadzadeh, J. Mol. Liq., 2016, 223, 1151-1157.

[44] M. Yoosefian, N. Etminan, RSC Adv., 2016, 6, 64818-6825.

[45] M. Yoosefian, H. Karimi-Maleh, A.L. Sanati, J. Chem. Sci., 2015, 127, 1007-1013.

[46] M. Yoosefian, Z. Ansarinik, N. Etminan, J. Mol. Liq., 2016, 213, 115121.

[47] (a) N. Etminan, M. Yoosefian, H. Raissi, M. Hakimi, J. Mol. Liq., 2016, 214, 313-318; (b) R.V. Kupwade, J. Chem. Rev., 2019, 1, 99-113.

[48] M. Yoosefian, A. Mola, J. Mol. Liq., 2015, 209, 526-530.

[49] S. Miertus, E. Scrocco, J. Tomasi, J. Chem. Phys., 1981, 55, 117-129.

[50] N.M. O'Boyle, A.L. Tenderholt, K.M. Langner, J. Comput. Chem., 2008 , 29, 839-845.

[51] M. Frisch, G. Trucks, H. Schlegel, G. Scuseria, M. Robb, J. Cheeseman, et al., Gaussian Inc, Wallingford, CT, 2004.

[52] S. Gunasekaran, R.A. Balaji, S. Kumeresan, G. Anand, S. Srinivasan, Can. J. Anal. Sci. Spectro., 2008, 53, 149-162.

[53] I. Fleming, Frontier Orbitals, Organic Chemical Reactions, John Wiley and Sons, New York, 1976.

[54] R.G. Pearson, J. Am. Chem. Soc., 1985, 107, 6801-6806.

[55] R.G. Pearson, Dowden, Hutchinson, Ross, Stroudsburg, PA, 1973.

How to cite this manuscript: Mehri Deilam, Ashraf Sadat Ghasemi, Fereydoun Ashrafi. "Study of bendamustine anticancer drug in gaseous and in a few selected liquid solvents phases using functional density theory (DFT)". Eurasian Chemical Communications, 2019, 518-526. 